Original Article

\title{
Multicenter survey analysis of satisfaction according to actual utilization of rehabilitation services
}

\author{
Byoung-Hwan Oh, PT, MS ${ }^{1)}$, Sung-Hyoun Cho, PT, PhD²), Jong-Won Lee, PT, PhD ${ }^{3)}$, \\ Chan-Woo Nam, PT, MS ${ }^{1 *}$ \\ 1) Department of Physical Therapy, College of Rehabilitation Science, Daegu University: \\ 201 Daegudae-ro, Gyeongsan-si, Gyeongsangbuk-do, Republic of Korea \\ 2) Department of Physical Therapy, Nambu University, Republic of Korea \\ 3) Department of Physical Therapy, Seoyeong University, Republic of Korea
}

\begin{abstract}
Purpose] Most such studies have been limited to evaluating the muscle activity patterns of lower extremity muscles using surface electromyograms, but studies of muscle activity patterns according to chair types are lacking. [Subjects and Methods] In the present study, 52 university students in their 20s (26 males, 26 females) were selected in order to analyze the activity of five lower extremity muscles (vastus lateralis, rectus femoris, vastus medialis, tibialis anterior, and gastrocnemius). The selected subjects had sufficient muscle strength for the experiment and normal joint range of motion. [Results] To examine the sociodemographic variables of the study subjects, the frequencies and percentages of individual items were presented as follows Table 3. [Conclusion] Differences in lower extremity muscle activity levels during standing and sitting were identified, and differences between genders were also seen. There was no significant difference in lower extremity muscle activity levels between use of fixed and wheeled chairs.

Key words: Sit to stand, Electromyograms, Muscle activity
\end{abstract}

(This article was submitted Sep. 30, 2015, and was accepted Nov. 17, 2015)

\section{INTRODUCTION}

Elementary, middle, and high school students in South Korea study and take examinations while seated for long hours in a chair. However, working long hours seated in a chair is a recent activity in human history to which our bodies have not yet fully adapted ${ }^{1)}$. To analyze this condition, previous studies mainly compared the time required to perform a sit to stand (STS) motion and the force and pressure applied to the lower extremity during the motion ${ }^{2}$; these studies reported that stroke patients took more time to stand, bent the torso more, and took more time to straighten the torso, compared to healthy elderly persons ${ }^{3)}$.

Restriction of STS motion is closely related to decreased balance ability ${ }^{4}$, and may also affect gait ability; restricted STS motion characteristically requires an extended time to perform, including lateral or medial displacement of the center of mass, and asymmetric weight bearing. However, use of the upper extremities for STS motion does not significantly reduce loads on lower extremity joints but only helps maintain balance ${ }^{5)}$. Among factors that interfere with STS motion, the height of the chair has the most critical effect ${ }^{6}$. STS motions are functional tasks performed many times each day and require lower extremity muscle strength and dynamic balance.

Muscle strength evaluation through the performance of STS motion can be conducted simply at home or in the clinic, and is a very useful and easy method for assessment of lower extremity strength ${ }^{7)}$ and functional conditioning ${ }^{8)}$, without a

\footnotetext{
*Corresponding author. Chan-Woo Nam (E-mail: somamir99@naver.com)

(C)2016 The Society of Physical Therapy Science. Published by IPEC Inc.

This is an open-access article distributed under the terms of the Creative Commons Attribution Non-Commercial No Derivatives (by-nc-nd) License $<$ http://creativecommons.org/licenses/by-nc-nd/4.0/>.
} 
Table 1. General characteristics of the subjects $(\mathrm{N}=52)$

\begin{tabular}{lccc}
\hline Category & Male $(\mathrm{n}=26)$ & Female $(\mathrm{n}=26)$ & Total $(\mathrm{N}=52)$ \\
\hline Age $(\mathrm{yrs})$ & $21.1 \pm 1.8$ & $20.5 \pm 1.2$ & $20.8 \pm 1.6$ \\
Height $(\mathrm{cm})$ & $174.9 \pm 4.3$ & $163.7 \pm 3.8$ & $169.3 \pm 6.9$ \\
Weight $(\mathrm{kg})$ & $69.2 \pm 10.1$ & $57.4 \pm 8.0$ & $63.3 \pm 10.8$ \\
BMI $\left(\mathrm{kg} / \mathrm{m}^{2}\right)$ & $22.6 \pm 3.1$ & $21.3 \pm 2.6$ & $22.0 \pm 2.9$ \\
\hline
\end{tabular}

M2.0: Mean \pm SD, BMI: body mass index

requirement for special training or equipment. In addition, horizontal moment STS motion involves movement of the center of gravity at the rear, requires relative balance control ${ }^{9)}$, and is used to evaluate functional movement ability ${ }^{10)}$.

The velocity of the center of gravity and lower extremity joint moment are important factors for evaluation of STS motion, and are used to evaluate the effects of angular joint motion in a given direction during the entire cycle of performance of a linear motion by the body ${ }^{11)}$. Studies of STS motion are largely conducted using three different methods: motion analysis based on kinematic systems; muscle activity pattern analysis based on electromyogram (EMG) signals; and methods that include kinetic analysis using force plates ${ }^{12}$. Among these, studies using electromyograms can measure how muscle energy supports the body against gravity, how muscles act to achieve targeted movements, and how much muscles rest in both healthy individuals and subjects with various diseases ${ }^{13)}$. However, most such studies have been limited to evaluating muscle activity patterns of lower extremity muscles using surface electromyograms ${ }^{14)}$, and studies of muscle activity patterns according to chair types are lacking. Because studies on STS evaluation are also lacking in South Korea, the present study was conducted to examine the effects of STS motion on lower extremity muscle activity according to chair type.

\section{SUBJECTS AND METHODS}

The present study was conducted in the exercise therapy room of $\mathrm{N}$ university located in Gwangju. The study was designed to analyze changes in the activity of lower extremity muscles, including the vastus medialis, rectus femoris, vastus lateralis, gastrocnemius, and the tibialis anterior, according to chair types by using EMG. The participants provided written informed consent in accordance with the ethical standards of the Declaration of Helsinki.

For the present study, 52 healthy students (26 males, 26 females) in their 20 s at N University in Gwangju were selected. The subjects had sufficient muscle strength for the experiment and normal joint range of motion.

The mean age of males was $21.10 \pm 1.86$ years, the mean height was $174.96 \pm 4.34 \mathrm{~cm}$, the mean weight was $69.23 \pm$ $10.11 \mathrm{~kg}$, and the body mass index (BMI) was $22.61 \pm 3.15 \mathrm{~kg} / \mathrm{m}^{2}$. The mean age of females was $20.54 \pm 1.27$ years, the mean height was $163.77 \pm 3.86 \mathrm{~cm}$, the mean weight was $57.46 \pm 8.09 \mathrm{~kg}$, and the BMI was $21.39 \pm 2.63 \mathrm{~kg} / \mathrm{m}^{2}(\mathrm{p}>0.05)($ Table 1$)$.

EMGs show invisible muscle activity during motion and EMG devices measure the electric signals generated by muscles during movement ${ }^{15)}$. A surface EMG (WEMG-8; LXM5308, Laxtha Inc., Korea) was used to measure the muscle activity of the vastus lateralis, the rectus femoris, the vastus medialis, the tibialis anterior, and the gastrocnemius; $112223 \mathrm{H}$ Electrodes were used. The sampling rate of signals was set for $1,024 \mathrm{~Hz}$, and $60-\mathrm{Hz}$ notch filters were used for sampling. A real-time analysis program (TeleScan; Laxtha Inc.) was used for EMG signal storage and processing.

All subjects were instructed to sit on a chair with no armrest that could be adjusted in height according to leg length, so that the midpoint of the hip was located at the end of the chair with the hip joint flexed at $90 \times$. To ensure consistent measurement postures for all subjects, the height of the chair was set so that the knee joint angle was at $90^{\circ}$. Because the hip joint is separated from the chair by the thickness of the tissues of the buttocks, the knee joint angle becomes $90^{\circ}$ when the height of the chair is approximately $80 \%$ of the knee joint ${ }^{16,17)}$. To avoid compensation for movements using the arms while preventing resistance, subjects crossed their arms on the chest. The subject was instructed to perform movements using only the trunk and the lower extremities while placing the feet at shoulder width. Sitting was used as the starting position and the subject performed 3 sets of STS motion for $5 \mathrm{~s}$ per set. To prevent muscle fatigue, the subject was instructed to rest for $10 \mathrm{~s}$ after each set. Electrodes were attached to all the muscles and the experimental method was explained to the subjects. Before measurement, the wires connecting the electrodes and the EMG were neatly organized and signals appearing on the screen were observed to ensure that movement noise would not be generated.

Before attaching the electrodes, the hair on the skin at the attachment sites was removed and the attachment sites were cleaned using alcohol gauze to minimize skin resistance. A ground electrode was attached to the C7 spinous process. To eliminate noise due to wire movements, the wires were fixed using Kinesio tape (Nippon Sigmax Co., Benefact, Japan), and in cases where noise was generated, the causes were eliminated before attaching the electrode. The distance between any two electrodes was maintained at $2 \mathrm{~cm}$ and the electrodes were attached parallel to the direction of muscle fibers. Surface electrodes were attached according to changes in the locations of muscle bellies during contraction (Table 2).

EMG signals were measured from the time when the researcher gave the command to "Start" to the point when the buttocks left the chair; the activity of the lower extremity muscles was measured during STS motion 3 times in $5 \mathrm{~s}$. The \%MVIC (Maximum Voluntary Isometric Contraction) of muscle activity, using the maximum isometric contraction value of one muscle measured during a standing motion as a reference value, based on the mean muscle signal quantity measured during 
Table 2. Electrode placement for detection of muscle activation

\begin{tabular}{|c|c|}
\hline Muscle & Electrode placement \\
\hline 1) Vastus medialis & $\begin{array}{l}\text { The distance along a line from the superior internal side of the patella to the anterior } \\
\text { medial iliac spine, starting from the patella }\end{array}$ \\
\hline 2) Rectus femoris & $\begin{array}{l}\text { The distance along a line from the superior medial side of the patella to the anterior } \\
\text { inferior iliac spine, starting from the patella }\end{array}$ \\
\hline 3) Vastus lateralis & $\begin{array}{l}\text { The distance along a line from the superior lateral side of the patella to the anterior } \\
\text { superior iliac spine, starting from the patella }\end{array}$ \\
\hline 4) Gastrocnemius & $\begin{array}{l}\text { The percentage distance from the medial side of the popliteus cavity to the medial } \\
\text { side of the Achilles tendon insertion, starting from the tuberosity of the tibia }\end{array}$ \\
\hline 5) Tibialis anterior & $\begin{array}{l}\text { The percentage distance from the tuberosity of the tibia to the medial malleolus line, } \\
\text { starting from the tuberosity of the tibia }\end{array}$ \\
\hline
\end{tabular}

3 seconds, excluding the first and last second, was used in data analysis ${ }^{18)}$.

The \%MVIC method shows muscle activity levels in certain motions as percentages, using the value obtained during the maximum isometric contraction of each muscle as a reference. In the present study, \%MVIC values were used as maximum isometric contraction values. To obtain the maximum torque values of individual muscles, the MVIC values of the vastus medialis (VM), rectus femoris (RF), vastus lateralis (VL), gastrocnemius (GC), and tibialis anterior (TA) were obtained with the knee joint flexed at $90^{\circ}$ in a sitting position. For muscle activity analysis, the MVIC was evaluated with the root mean square (RMS) value of the knee extensor muscle measured when muscle contracted for $5 \mathrm{~s}$ in the manual muscle testing posture.

The muscle activity levels of individual muscles according to chair types were measured and processed into the RMS, and individual muscle activity levels during the 3 middle seconds were converted into percentages (\%MVIC-RMS). Thereafter, using normalized muscle activity values according to chair types, the ratios of activity of the VM, RF, VL, GC, and TA were calculated.

Means and standard deviations for physical characteristics by group and measured data by variables were calculated. The data from the results of the present study were processed using the SPSS ver. 18.0 statistical program. Independent t-tests were conducted to verify differences in the values between chair types and between genders. Paired t-tests were conducted to analyze differences in the values between the standing group and sitting group, and the statistical significance level $\alpha$ was set to 0.05 .

\section{RESULTS}

The frequencies and percentages of individual sociodemographic variables of the study subjects are presented in Table 3 . The genders of the study subjects were balanced, with 26 males and 26 females. For age distribution, 20 years was highest at $55.8 \%$, followed in order by 21,24 or more, 19 , and 23 . Most subjects $(78.8 \%)$ thought they were healthy, followed in order by those who thought they were moderately healthy, and those who thought they were weak.

There were more nonsmokers (75\%) than smokers. The right foot was dominant in 92.3\%. Those Most (69.2\%) drank 1-2 times per week, followed by those who did not drink, and those who drank at least 3 times per week. Regarding family composition, subject + parents was highest at $76.9 \%$, followed in order by subject + parents + siblings, and the subject alone. Those with monthly incomes at least 300,000 won were highest at 48.0\%, followed by those with 200,000-290,000 won, and those with 100,000-190,000 won.

Using a fixed chair during standing, the muscle activity levels of the VM, RF, and VL increased more in females than in males, and the activity levels of the GC and TA decreased more in females than in males. During sitting, the activity levels of the VM, RF, and VL increased more in females than in males, and the activity levels of the GC and TA decreased more in females than in males. Using a wheeled chair during standing, the activity levels of the VM, RF, and VL increased more in females than in males, and the activity levels of the GC and TA decreased more in females than in males. During sitting, the activity levels of the VM, RF, VL, and GC increased more in females than in males, and the activity levels of the TA decreased more in females than in males.

\section{DISCUSSION}

STS motions are the functional tasks most commonly required in daily living. STS motions also precede functional activities such as walking ${ }^{19)}$, and have become a practical evaluation method frequently used in technical and rehabilitation studies ${ }^{20)}$. When STS motions were performed using different chair types, there was no significant difference in the muscle activity levels of the VM, RF, VL, GC, and TA between fixed and wheeled chairs ( $>0.05)$. The lateral flexion of the trunk toward the lower extremities and weight bearing by the lower extremities are expected to increase when subjects perform STS motions on a wheeled chair, so that the activity levels of the RF, VL, and VM would increase compared to when subjects 
Table 3. Sociodemographic variables of the subjects $(\mathrm{N}=52)$

\begin{tabular}{|c|c|c|c|}
\hline Category & Division & Frequency & Percentage $(\%)$ \\
\hline \multirow{2}{*}{ Gender } & Man & 26 & 50.0 \\
\hline & Woman & 26 & 50.0 \\
\hline \multirow{5}{*}{ Age } & 19 & 4 & 7.7 \\
\hline & 20 & 29 & 55.8 \\
\hline & 21 & 12 & 23.1 \\
\hline & 23 & 2 & 3.8 \\
\hline & More than 24 years of age & 5 & 9.6 \\
\hline \multirow{3}{*}{ Health condition } & Health & 41 & 78.8 \\
\hline & Moderate & 10 & 19.3 \\
\hline & Weakness & 1 & 1.9 \\
\hline \multirow{2}{*}{ Smoke } & Non-smoking & 39 & 75.0 \\
\hline & Smoke & 13 & 25.0 \\
\hline \multirow{2}{*}{ Dominant foot } & Left-foot & 4 & 7.7 \\
\hline & Right Foot & 48 & 92.3 \\
\hline \multirow{3}{*}{ Alcohol consumption } & No alcohol & 13 & 25.0 \\
\hline & $1-2$ times a week & 36 & 69.2 \\
\hline & More than three times a week & 3 & 5.8 \\
\hline \multirow{3}{*}{ Family members } & myself & 2 & 3.8 \\
\hline & myself + parents & 40 & 76.9 \\
\hline & myself + parents + siblings & 10 & 19.3 \\
\hline \multirow{4}{*}{ Salary } & 90,000 or less & 12 & 23.1 \\
\hline & $¥ 100,000-190,000$ & 3 & 5.8 \\
\hline & $¥ 200,000-290,000$ & 12 & 23.1 \\
\hline & $¥ 300,000$ or more & 25 & 48.0 \\
\hline Total & & 52 & 100.0 \\
\hline
\end{tabular}

perform STS motions on a fixed chair.

The present study showed that when STS motions were performed on a wheeled chair, the activity levels of the GC and TA during standing and sitting were not significantly different between males and females ( $>0.05)$, while the activity levels of the VM, RF, and VL were significantly different between males and females $(p<0.05)$. Khemlani ${ }^{21)}$ examined the activation patterns of the lower extremity muscles of males and females during standing with both feet placed symmetrically and reported that the GC and soleus were not activated at the beginning of STS motion. This is consistent with the results of the present study, with no significant difference in the activity of the GC during standing and sitting between males and females.

There was no significant difference in muscle activity levels during STS motions on stable bearing surfaces between fixed and wheeled chairs; future studies conducted under controlled conditions with unstable bearing surfaces and a variety of additional dependent variables should provide basic data on lower extremity muscle activity levels when different chair types are used. Future studies should have adequate test power, with more study subjects and different evaluation tools.

Since most prior studies only focused on the analysis of STS motion and chair types, the present study was conducted to analyze muscle activity levels during STS motion according to chair types and genders. The present study was conducted with 52 experimental subjects to examine lower extremity muscle activity levels during STS motion on fixed and wheeled chairs using an EMG.

When STS motions were assessed on different types of chairs by gender, there were significant differences in muscle activity levels of the RF and VL during standing using a fixed chair; during sitting, there were significant differences in activity levels of the $\mathrm{VM}, \mathrm{RF}$, and $\mathrm{VL}(\mathrm{p}<0.05)$. In standing using a wheeled chair, there were significant differences in the activity levels of the RF and VL; during sitting, there were significant differences in the activity levels of the VM, RF, and $\mathrm{VL}(\mathrm{p}<0.05)$.

The present study showed differences in lower extremity muscle activity levels during standing and sitting, and differences between genders were also observed. There was no significant difference in lower extremity muscle activity levels between use of fixed and wheeled chairs. Future studies should have adequate test power for kinematic analysis of STS motion by utilizing more study subjects and different evaluation tools. 


\section{REFERENCES}

1) Jang EM, Yoo WG: Comparison of the gluteus medius and rectus femoris muscle activities during natural sit-to-stand and sit-to-stand with hip abduction in young and older adults. J Phys Ther Sci, 2015, 27: 375-376. [Medline] [CrossRef]

2) Chou SW, Wong AM, Leong CP, et al.: Postural control during sit-to stand and gait in stroke patients. Am J Phys Med Rehabil, 2003, 82: 42-47. [Medline] [CrossRef]

3) Choi Y, Her JG, Ko J, et al.: The effects of chair height and foot position on chronic stroke patients' sit-to-walk movement. J Phys Ther Sci, 2013, 25: 431-435. [CrossRef]

4) Maeda N, Kato J, Itotani K, et al.: Relationship between sit-to-stand (STS) motion characteristics and walking ability in stroke patients. Gait Posture, 2012, 36: 57-58. [CrossRef]

5) Lomaglio MJ, Eng JJ: Muscle strength and weight-bearing symmetry relate to sit-to-stand performance in individuals with stroke. Gait Posture, 2005, 22: 126-131. [Medline] [CrossRef]

6) Scholz JP, Reisman D, Schöner G: Effects of varying task constraints on solutions to joint coordination in a sit-to-stand task. Exp Brain Res, 2001, 141: 485-500. [Medline] [CrossRef]

7) Hardy R, Cooper R, Shah I, et al.: Is chair rise performance a useful measure of leg power? Aging Clin Exp Res, 2010, 22: 412-418. [Medline] [CrossRef]

8) Janssen WG, Bussmann HB, Stam HJ: Determinants of the sit-to-stand movement: a review. Phys Ther, 2002, 82: 866-879. [Medline]

9) Mong Y, Teo TW, Ng SS: 5-repetition sit-to-stand test in subjects with chronic stroke: reliability and validity. Arch Phys Med Rehabil, 2010, 91: 407-413. [Medline] [CrossRef]

10) Butler AA, Menant JC, Tiedemann AC, et al.: Age and gender differences in seven tests of functional mobility. J Neuroeng Rehabil, 2009, 6: 31-35. [Medline] [CrossRef]

11) Yu B, Holly-Crichlow N, Brichta P, et al.: The effects of the lower extremity joint motions on the total body motion in sit-to-stand movement. Clin Biomech (Bristol, Avon), 2000, 15: 449-455. [Medline] [CrossRef]

12) Kim J, Gong W, Hwang B: Effects of lumbar resistance and stabilization complex exercises on extremity muscle strength and endurance of normal adults. J Phys Ther Sci, 2011, 23: 645-649. [CrossRef]

13) Cram JR: The history of surface electromyography. Appl Psychophysiol Biofeedback, 2003, 28: 81-91. [Medline] [CrossRef]

14) Chae WS: Electromyographic Analyses of the effects of different foot positions during exercise on a stair-climbing machine. Korean J Sport Biomech, 2005, 15: 207-219. [CrossRef]

15) De Luca CJ, Gilmore LD, Kuznetsov M, et al.: Filtering the surface EMG signal: movement artifact and baseline noise contamination. J Biomech, 2010, 43: 1573-1579. [Medline] [CrossRef]

16) Schenkman M, Berger RA, Riley PO, et al.: Whole-body movements during rising to standing from sitting. Phys Ther, 1990, 70: 638-648, discussion 648-651. [Medline]

17) Sriwarno AB, Shimomura Y, Iwanaga K, et al.: The effects of heel elevation on postural adjustment and activity of lower-extremity muscles during deep squatting-to-standing movement in normal subjects. J Phys Ther Sci, 2008, 20 : 31-38. [CrossRef]

18) Cifrek M, Medved V, Tonković S, et al.: Surface EMG based muscle fatigue evaluation in biomechanics. Clin Biomech (Bristol, Avon), 2009, 24: 327-340. [Medline] [CrossRef]

19) Cahill BM, Carr JH, Adams R: Inter-segmental co-ordination in sit-to-stand: an age cross-sectional study. Physiother Res Int, 1999, 4: 12-27. [Medline]

20) Kerschan-Shindl K, Uher E, Kainberger F, et al.: Long-term home exercise program: effect in women at high risk of fracture. Arch Phys Med Rehabil, 2000, 81: 319-323. [Medline] [CrossRef]

21) Khemlani MM, Carr JH, Crosbie WJ: Muscle synergies and joint linkages in sit-to-stand under two initial foot positions. Clin Biomech (Bristol, Avon), 1999, 14: 236-246. [Medline] [CrossRef] 